\title{
Pilot Study on Malnutrition and DNA Damage in Patients with Newly Diagnosed Gastrointestinal Tumors: Is DNA Damage Reversible by Early Individualized Nutritional Support?
}

\author{
Liana M. Bergholz ${ }^{1,4}$, Peter Grimminger ${ }^{2}$, Elsa Dikeoulia ${ }^{1}$, Heidi Rossmann ${ }^{3}$, Arndt Weinmann ${ }^{1}$, Markus Möhler ${ }^{1}$, Tim \\ Zimmermann $^{1}$, Matthias M. Weber ${ }^{1}$, Peter R. Galle ${ }^{1}$, Bernd Kaina ${ }^{4^{*}}$, Anca Zimmermann ${ }^{1,5^{*}}$
}

\author{
1) $1^{\text {st }}$ Clinic and Polyclinic of \\ Internal Medicine, University \\ Medical Center Mainz; \\ 2) Clinic of General, Visceral \\ and Transplantation Surgery, \\ University Medical Center \\ Mainz; \\ 3) Institute of Clinical \\ Chemistry and Laboratory \\ Medicine, University Medical \\ Center, Mainz; \\ 4) Institute of Toxicology, \\ University Medical Center, \\ Mainz; \\ 5) Medical Clinic 2, \\ Dept. of Diabetology and \\ Endocrinology, Clinic of \\ Worms, Germany
}

\section{ABSTRACT}

Background \& Aims: Nutritional support (NS) in patients with malignancies and malnutrition improves outcome and treatment tolerance. The underlying mechanisms are not completely understood. We aimed to investigate for the first time the influence of an early individualized NS in newly diagnosed patients with gastrointestinal/hepato-pancreatic malignancies and malnutrition on DNA damage, oxidative stress and subclinical inflammation.

Methods: This prospective case-control study included 43 patients with newly diagnosed malignancies and malnutrition. At baseline (F0), we documented patients' data, oncological diagnosis, comorbidities, alcohol/ nicotine consume. Nutritional parameters, DNA damage [histone-variant $\mathrm{H} 2 \mathrm{AX}$ phosphorylated on the 139-serine residue $(\gamma$-H2AX) foci/cell], oxidative status, subclinical inflammation were measured. During diagnostic workup, patients received an individualized NS, and got a follow-up before the start of treatment (F1), $(n=21)$. Healthy controls $(n=21)$ were included for comparison of DNA damage at baseline.

Results: $\gamma$-H2AX-values at baseline were higher than in controls $(\mathrm{p}<0.001)$ and higher than after the NS at F1 $(p=0.011)$. Patients with severe gastrointestinal symptoms (SGS) had higher baseline foci compared to patients with mild gastrointestinal symptoms $(\mathrm{MGS})$ at F0 $(\mathrm{p}<0.001)$ and showed a stronger decrease of DNA damage under NS ( $\mathrm{p}=0.002)$. Laboratory data were stable, with tendential reduction in oxidative stress, without progression of subclinical inflammation. The number of $\gamma$-H2AX foci did not differ among patients divided by sex, age, nicotine or alcohol intake or the presence of distant metastases.

Conclusion: Increased baseline DNA damage in patients with newly diagnosed tumors and malnutrition decreased under pretherapeutic NS, independent of other known genotoxic factors. This contributes towards understanding the positive effects of early NS in cancer management.

Key words: DNA damage - malignancy - malnutrition - oxidative stress - nutritional support.

Abbreviations: ATM: ataxia-teleangiectasia; BMI: body mass index; DSB: double strand break; ESPEN: European Society for Clinical Nutrition and Metabolism; F0: baseline; F1: follow-up before the start of treatment; GPX: glutathione peroxidase; hsCRP: high sensitive C reactive protein; MGS: mild gastrointestinal symptoms; MN: malnutrition; NRS: nutritional risk score; NS: nutritional support; PAI-1: plasminogen activator inhibitor 1; PBLC: peripheral blood lymphoid cells; ROS: reactive oxygen species; SGS: severe gastrointestinal symptoms; SOD: superoxid-dismutase; TAC: total antioxidant capacity; TPN: total parenteral nutrition; $\gamma$-H2AX: histone-variant H2AX, phosphorylated on the 139-serine residue.

\section{INTRODUCTION}

Patients with gastrointestinal malignancies are at an increased risk for malnutrition $(\mathrm{MN})$. The improvement of the nutritional status by sufficient intake of energy and nutrients, adapted to the individual patients' needs, is a central task of guideline-based nutritional intervention, aiming to stop the weight loss, to preserve the treatment tolerance, to reduce the adverse effects of therapy and to improve the quality of life [1]. Surgery of the gastrointestinal tract should be postponed for some weeks until nutritional stabilization in patients at high nutritional risk, presenting either with a weight loss $>10-15 \%$ in the last six months, a body mass index (BMI) $<18.5 \mathrm{~kg} / \mathrm{m}^{2}$ or a serum albumin $<30 \mathrm{~g} / \mathrm{dl}$, without hepatic or renal dysfunction [2].

Malignancies and their treatment lead to severe physiological and biochemical alterations. Malignancy- 
associated MN often progresses to tumor cachexia, with complex interactions between proinflammatory cytokines and oxidative stress. An early, individualized nutritional intervention with counselling and oral, enteral or parenteral supplementary or complete caloric provision is established as an obligate step in the surgical, radiative or chemotherapeutical therapy of patients with malignancies and has been shown to improve functional status, survival and quality of life [3]. Glutamin, n-3 fatty acids and pro-/prebiotics modulated the gastrointestinal toxicity under chemotherapy [4]. An effective preoperative nutritional support (NS) with standard or immune-enhancing enteral solutions significantly increased the total antioxidative capacity in patients with gastrointestinal malignancies and $\mathrm{MN}$ [5]. A postoperative total parenteral nutrition (TPN) reduced oxidative stress in patients with intestinal and pancreatic tumors and MN [6]. On the other hand, $\mathrm{MN}$ was associated with increased mortality and poor tolerance to palliative chemotherapy in older patients with colorectal carcinomas [7].

The mechanisms by which nutritional intervention improves treatment tolerance, quality of life, reduces surgeryassociated complications and improves survival are not totally explained by the increase in body weight and muscle mass and are up to now poorly investigated and not completely understood.

Malnutrition and nutritional intervention could influence DNA damage and repair. Increased reactive oxygen species (ROS)-status could contribute to DNA damage in healthy tissues. DNA damage is expressed by double strand breaks (DSBs), which are cytotoxic and can lead to chromosomal aberrations [8]. Double strand breaks lead to an early activation of phosphatidylinositol 3-kinase (PI3K)-like proteinkinase ataxia-telangiectasia-mutated (ATM) protein [9]. An important target protein of ATM is the histone-variant $\mathrm{H} 2 \mathrm{AX}$, which is phosphorylated on the 139-serine residue $(\gamma-\mathrm{H} 2 \mathrm{AX})$ following DSBs and represents a specific marker for DNA damaging exposure [10]. The immunofluorescence-based method for visualizing $\gamma$-H2AX-foci in lymphocytes from peripheral blood is a highly sensitive method for the detection and quantification of DSBs, which are increased in tumor tissues, chronic inflammatory diseases, ischemia-reperfusion-processes or following radiation [8, 11-14].

Recent data point out to a relation between DSBs and malignancy progression in bladder, breast and colon cancer [15-18]. Beside these findings, chronic hypoxemia, heart failure, smoking and alcohol consumption are further factors to increase DSBs [19-21].

There are few literature data, suggesting a reduction in DNA damage and increase of antioxidant capacity by iron supplementation, fruit juice and carotenoid-rich vegetables consumption, but no data analyzing the impact of nutritional intervention on these parameters in patients with newly diagnosed malignancies [22-24].

Therefore, we aimed to investigate for the first time the influence of an early individualized nutritional intervention in newly diagnosed patients with gastrointestinal cancer and MN on DNA damage, oxidative stress and subclinical inflammation.

\section{METHODS}

This was a monocentric prospective case-control study. We screened for MN 70 patients with gastrointestinal and hepatopancreatic tumors, referred to the Medical Clinic and Polyclinic or the Clinic for General, Visceral and Transplantation Surgery of the University Medical Center Mainz, Germany. All included patients and controls provided written informed consent. The study was conducted according to the ethical guidelines of the Declaration of Helsinki and was approved by the Ethics Committee of the Rhineland-Palatinate. Inclusion criteria were: newly diagnosed malignancy without previous treatment, age $>18$ years, $\mathrm{MN}$, willingness to participate in the study. Malnutrition was defined, according to the ESPEN diagnostic criteria, as a weight loss of more than $10 \%$ (indefinite of time) or more than $5 \%$ over the last three months and a BMI $<20 \mathrm{~kg} / \mathrm{m}^{2}$ or $<22 \mathrm{~kg} / \mathrm{m}^{2}$ in patients under or above the age of 70, respectively [25]. Exclusion criteria were: psychiatric comorbidity, recurrent cancer, history of treatment for another cancer in the past five years, unstable condition, immune deficiency disorders (e.g. HIV positive) and patients who had commenced treatment for the gastrointestinal tumor in another center. All included patients provided written informed consent.

As a control group for comparison of DNA damage, we included 21 healthy volunteers from the University Medical Center Mainz, matched with the patients with respect to age, gender, nicotine and alcohol consumption. They provided also written informed consent for anonymized documentation of their data: age, gender, height $(\mathrm{cm})$, weight $(\mathrm{kg}), \mathrm{BMI}\left(\mathrm{kg} / \mathrm{m}^{2}\right)$, nicotine and alcohol consumption and for the collection of 5 ml EDTA-blood.

Patients were screened for malnutrition by our Nutritional Team using the Nutritional Risk Score (NRS) questionnaire. This is an appropriate tool to screen and identify patients at increased risk for $\mathrm{MN}$, assessing the dynamics of unintended weight loss and comorbidities [26]. Patients with NRS values $\geq 3$ were considered at risk for $\mathrm{MN}$, received a detailed nutritional anamnesis and were subjected to an individualized nutritional counselling. This time point was the baseline (F0) evaluation for the patients meeting the criteria for $\mathrm{MN}$ (F0). During the 3-4 weeks required for diagnostic workup with histological diagnosis and appropriate staging and up to the start of treatment (F1) (surgery or neoadjuvant chemotherapy or radiation), patients received a caloric supplementation up to the optimum of $25-30 \mathrm{kcal} / \mathrm{kg}$ body weight, according to their individual needs and current guidelines, by fully balanced oral NS and/or parenteral nutrition [27]. After these 3-4 weeks and 1-3 days before treatment start, patients with MN were reevaluated $(\mathrm{F} 1)$.

At F0 we documented for each patient with $\mathrm{MN}$ : age, gender, height $(\mathrm{cm})$, weight $(\mathrm{kg}$; measured in the morning, with light clothes and without shoes, recorded to the nearest $0.1 \mathrm{~kg})$, BMI ( $\mathrm{kg} / \mathrm{m}^{2}$, Seca 702 Hamburg/Germany), medical history, physical examination, oncological diagnosis (tumor dignity, localization), comorbidities (diabetes mellitus, coronary heart disease, metabolic syndrome), nicotine intake (continued in the present, defined as pack-years), alcohol consumption (continued in the present, defined as $>30 \mathrm{~g} /$ day in 
men and $>20 \mathrm{~g} /$ day in women), family history for malignancies. Underweight was defined as BMI $<20$ or $22 \mathrm{~kg} / \mathrm{m}^{2}$ in patients younger and older than 70 years, respectively, as proposed by the European Society for Clinical Nutrition and Metabolism (ESPEN) [25].

The following measurements were performed from peripheral venous blood at the time points $\mathrm{F} 0$ and $\mathrm{F} 1$ : a) routine measurements for the nutritional and metabolic evaluation; $b$ ) measurement of DNA damage; c) measurement of oxidative stress and of subclinical inflammation.

The routine measurements for the nutritional and metabolic evaluation included total proteins, albumin, prealbumin, blood count, iron, ferritin, transferrin, transferrin saturation, vitamin B12, folic acid, zinc, selenium, 25-hydroxy-vitamin D.

The measurement of DNA damage by immunofluorescence out of peripheral blood lymphoid cells (PBLC) quantified the $\gamma$-H2AX (foci/cell) in the Institute for Toxicology of the University Medical Center Mainz, Germany [10, 28-30]. In short, the fresh EDTA venous blood samples were first incubated at $37^{\circ} \mathrm{C}$ for $1 \mathrm{~h}$ in the dark. $20 \mu \mathrm{l}$ blood of every sample was dropped on a microscope slide and was dispersed, helped by a second microscope slide, across the whole slide length, to make a blood smear. After 10 min drying, the blood smears were fixed with $0.5 \mathrm{ml}$ of $4 \%$ paraformaldehyde solution for $15 \mathrm{~min}$ at RT and then permeabilized with 1.5 $\mathrm{ml}$ ice-cold methanol:aceton solution $(7: 3)$ for $10 \mathrm{~min}$ at $-20^{\circ} \mathrm{C}$. One previously prepared blood smear (the positive control), containing irradiated blood (2 Gy), fixed with $4 \%$ paraformaldehyde as described before and stored in methanol at $-20^{\circ} \mathrm{C}$, was added to the blood smears of the patients. After the rehydration of all blood smear slides by washing them three times in PBS, the slides were incubated with $150 \mu$ l of blocking solution (PBS $+0.25 \%$ Triton-X100 + 10\% NGS) for $1 \mathrm{~h}$ at RT (dark and humid atmosphere). After a new wash in PBS, the slides were placed in 6-well plates and treated with $80 \mu \mathrm{l}$ of the first antibody solution (anti- $\gamma \mathrm{H} 2 \mathrm{AX}$, Millipore, diluted 1:1000 in PBS / $0.25 \%$ TritonX-100) and then placed overnight by $4^{\circ} \mathrm{C}$. Next day the slides were washed 2 times in PBS, 1 time in PBS "high salt” and again 1 time in PBS, and then incubated with $80 \mu \mathrm{l}$ of the second antibody solution (Alexa488 goat antimouse, Millipore, diluted 1: 500 in PBS $+0.25 \%$ TritonX) for $1 \mathrm{~h}$ at RT. After another four wash steps (as described above), one drop Vectashield mounting medium with DAPI, Vector, was dropped on every slide. They were then covered with coverslips and sealed with nail polish. The evaluation was carried out automatically using a Metafer microscope with Metafer4 Software (MetaSystems, Carl Zeiss, Jena), for about 500 cells per slide and per sample. The statistical evaluation of the foci gained by Metafer occurred semi-automatically (ImageJ/Fiji Software).

Measurement of oxidative stress included total antioxidant capacity (TAC) (colorimetric method, EDTA-plasma, PerOx TOS/TOC kit from Immundiagnostik, Ref KC5100, Germany), superoxid-dismutase (SOD) and glutathionperoxidase (GPX) from washed erythrocytes (colorimetric, RANSOD and RANSEL assays from Randox, United Kingdom. The measurements of subclinical inflammation included interleukin 6 (IL6) in serum (Cobas Elecsys 411 IL-6 kit, Roche Diagnostics, Germany), plasminogen activator inhibitor
1 (PAI-1) from citrate-plasma (TECHNOZYM ${ }^{\circledR}$ PAI-1 Antigen ELISA kit, Technoclone, Austria), high sensitive C reactive protein (hsCRP) (CRP Vario, Abbott Laboratories, Wiesbaden, Germany) in the Institute for Clinical Chemistry and Laboratory Medicine of the University Medical Center Mainz, Germany.

Further documented data at F1 were: weight $(\mathrm{kg}), \mathrm{BMI}(\mathrm{kg} /$ $\mathrm{m}^{2}$ ), physical examination, completion of oncological diagnosis (staging, histology).

Quantitative variables were expressed as means and standard deviations if they followed a normal distribution or as medians and quartiles otherwise. The distribution of continuous variables was assessed with the Shapiro Wilk test and quantile-quantile plots. If the data were normally distributed, we analyzed the equality of variances with the Levene's test and compared the independent samples using the unpaired t-test. Otherwise, the nonparametric Mann-Whitney U test was used. For comparisons of matched, dependent groups of continuous data, the paired t-test was used for data following the normal distribution; otherwise, the Wilcoxon signed-rank test was used. The Spearman correlation coefficient was employed to measure the relationships between the $\gamma$-H2AX (foci/cell) and clinical parameters, as well as laboratory data relevant for $\mathrm{MN}$, oxidative stress and subclinical inflammation. The $\mathrm{p}$ values < 0.05 were considered statistically significant. However, because this was a pilot study, the analyses were explorative and have to be interpreted as such. Statistical evaluation was performed with the statistical package IBM SPSS 23.0 for Windows.

\section{RESULTS}

From 70 patients with newly diagnosed gastrointestinal tumors, screened for MN with the NRS, 52 patients had an NRS $\geq 3$ (74\%). From the 52 patients, 43 fulfilled the diagnostic criteria for $\mathrm{MN}$ and were included in the study at baseline [25]. Only 21 patients of these could be evaluated at F1, due to the following reasons: 10 patients decided to start the treatment in their hometown and 12 patients accepted the nutritional counselling and support but refused the second blood measurement. The characteristics of patients and controls are given in Table I.

$\gamma$-H2AX (foci/cell) were identified using immunofluorescence microscopy in PBLCs and are shown as examples, as captured by the Metafer microscopy system, in Fig. 1A. $\gamma$-H2AX (foci/ cell) [F0 total, all patients; median $0.35(0.15-0.77)]$ were at baseline significantly higher than in controls [median 0.13 $(0.06-0.22) ; \mathrm{p}<0.001)]$ and significantly higher than after the individualized nutritional intervention at F1 [median 0.15 (0.090.32); $\mathrm{p}=0.011$ ]. Furthermore, the comparison of matched F0 values [in patients who received the follow-up measurement; $\mathrm{n}=21$; median 0.36 (0.21-0.52)] with $\mathrm{F} 1$ values, showed also a significant decrease under NS $(\mathrm{p}=0.013)$. F1 values dropped to levels comparable to controls (Fig. 1B). The individual values of $\gamma$-H2AX (foci/cell) at F0 ( $\mathrm{n}=21$, matched with F1), at F1 and in controls are depicted in Fig. 1C. All patients presented weight loss $>5 \%$ in the last three months or $>10 \%$ in an indefinite time. All of them complained about inappetence, which was defined as a mild gastrointestinal symptom (MGS). If patients also complained about repetitive nausea and vomiting, they were 
Table I. Characteristics of patients and controls

\begin{tabular}{|c|c|c|c|c|c|}
\hline & $\begin{array}{c}\text { Patients F0 } \\
n=43\end{array}$ & $\begin{array}{c}\text { Patients F1 } \\
n=21\end{array}$ & $\mathrm{p}(\mathrm{F} 0 / \mathrm{F} 1)$ & $\begin{array}{c}\text { Controls } \\
n=21\end{array}$ & $\begin{array}{c}\mathrm{p}(\mathrm{F} 0 / \\
\text { controls })\end{array}$ \\
\hline Age (years) & $64.7 \pm 10.7$ & $63.52 \pm 12.6$ & 0.61 & $62.55 \pm 12.77$ & 0.43 \\
\hline Gender male, $\mathrm{n}$ & 29 & 15 & 0.74 & 12 & 0.56 \\
\hline BMI $\left(\mathrm{kg} / \mathrm{m}^{2}\right)$ & $19.5 \pm 1.5$ & $19.8 \pm 1.4$ & 0.77 & $20.4 \pm 3.2$ & 0.55 \\
\hline Weight loss* & 43 & 21 & & & \\
\hline SGS ${ }^{* *}$ & 23 & 12 & 1.00 & & \\
\hline \multicolumn{6}{|l|}{ Malignancies, $\mathrm{n}$} \\
\hline Oesophageal & 18 & 9 & & & \\
\hline Gastric & 9 & 5 & & & \\
\hline Pancreatic & 11 & 4 & & & \\
\hline Hepatocellular & 5 & 3 & & & \\
\hline Metastasis & 25 & 11 & 0.79 & & \\
\hline Malignancies in family history & 10 & 4 & 0.76 & 3 & 0.40 \\
\hline \multicolumn{6}{|l|}{ Comorbidities, $\mathrm{n}$} \\
\hline Coronary artery disease & 3 & & & & \\
\hline Diabetes mellitus type 2 & 4 & & & & \\
\hline Smoking & 27 & & & 12 & 0.78 \\
\hline Alcohol consumption & 12 & & & 5 & 0.73 \\
\hline Nutritional risk score & $3.55 \pm 0.69$ & & & & \\
\hline Triceps thickness $(\mathrm{cm})$ & $8.0(6.0-13.5)$ & $9.5(7.8-14.7)$ & 0.82 & & \\
\hline Oral NS & 15 & 6 & 0.255 & & \\
\hline Parenteral NS & 23 & 12 & 0.782 & & \\
\hline Oral and parenteral NS & 5 & 3 & 0.762 & & \\
\hline
\end{tabular}

F0: baseline; F1: follow-up before the start of treatment; ${ }^{*}$ weight loss: $>5 \%$ over the last three months or $>10 \%$ in an indefinite time; ${ }^{* *}$ SGS: severe upper gastrointestinal symptoms: appetite loss, nausea, vomiting; NS nutritional support.

A

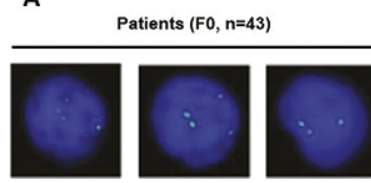

B
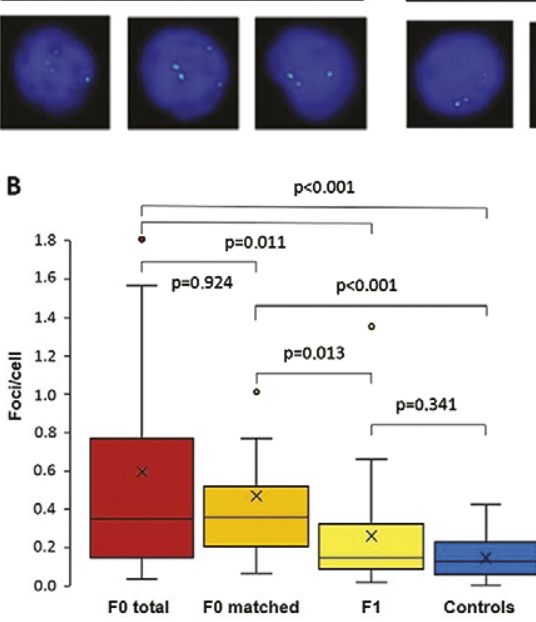

Patients (F1, n=21)

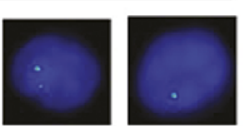

C

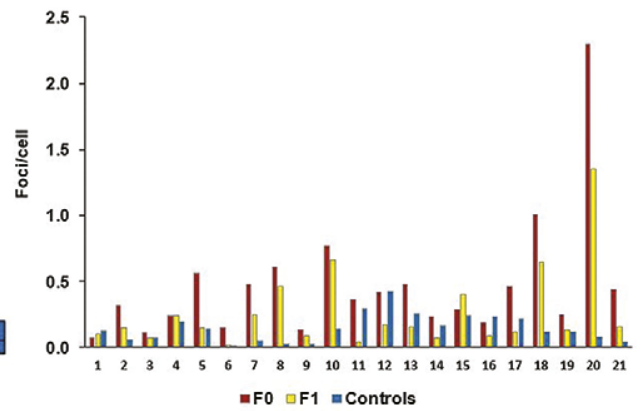

Fig. 1. Levels of $\gamma-\mathrm{H} 2 \mathrm{AX}$ foci/cell in patients at F0 (at baseline), F1 (after nutritional intervention) and in healthy controls. A: $\gamma$-H2AX foci/cell in patients at F0, F1 and controls. Foci were determined using immunofluorescence microscopy (Metafer microscopy system) in PBLCs ( $\mathrm{n}=500$ cells/sample). The images are examples of representative cells containing foci. B: $\gamma-\mathrm{H} 2 \mathrm{AX}$ foci/cell at baseline in all patients ( $\mathrm{F} 0$ total, $\mathrm{n}=43$, foci/cell $=0.35(0.15-0.77)$ ), at baseline in all patients which received follow-up (F0 matched, $\mathrm{n}=21$, foci/cell $=0.36(0.21-0.52)$ ), at follow-up $(\mathrm{F} 1, \mathrm{n}=21$, foci/cell $=0.15(0.09-0.32)$ ) and in controls (measured at baseline, $\mathrm{n}=21$, foci/cell $=0.13(0.06-0.22)$ ). Foci/cell are given as medians and interquartile ranges. Non-parametric tests for two independent samples (Mann-Whitney-Utest) were performed for intergroup comparisons and $p$-values $<0.05$ were considered significant. C: Individual values of the $\gamma$-H2AX foci/cell in patients at $\mathrm{F} 0$ ( $\mathrm{n}=21$, matched), at $\mathrm{F} 1$ and in controls. 
classified as suffering from severe gastrointestinal symptoms (SGS). Patients with SGS had higher median baseline foci values compared to patients with MGS at F0 [0.65 (0.46-1.34) vs. $0.15(0.10-0.23), \mathrm{p}<0.001]$ and only tendentially higher values at F1 [0.16 (0.15-0.60) vs. $0.09(0.07-0.18), \mathrm{p}=0.09]$. All patients with SGS had individual $\gamma$-H2AX (foci/cell) values $>0.30$ at baseline $(n=23)$, while all patients in the MGS group had values $<0.30(n=20)$ at this time point. Patients with SGS had a stronger decrease of DNA damage under the short individualized nutritional intervention compared to those with MGS [0.32 (0.11-0.44) vs. 0.06 (0.01-0.14), p=0.002], as shown in Fig 2. $\gamma$-H2AX (foci/cell) did not correlate with BMI at any time point. $\gamma$-H2AX (foci/cell) were not higher in patients with NRS scores $>3(n=19)$ compared to those with scores $=3(n=24)$ $(\mathrm{p}=0.831)$ and there was no correlation between the foci number and NRS-values (data not shown).

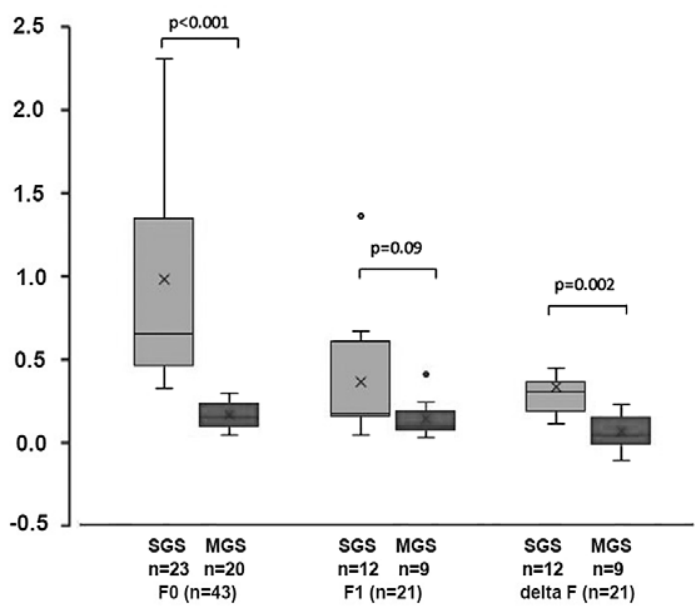

Fig. 2. $\gamma-\mathrm{H} 2 \mathrm{AX}$ foci/cell in patients with severe upper gastrointestinal symptoms (inappetence and nausea/ vomiting, SGS), compared to patients with mild gastrointestinal symptoms (inappetence only, MGS) at F0 [0.65 (0.46-1.34) vs. $0.15(0.10-0.23), \mathrm{p}<0.001]$; F1[0.16 (0.15-0.60) vs. 0.09 (0.07-0.18), p=0.09] and comparison of the change in foci/cell between F0 and F1 (deltaF), [0.32 (0.11-0.44) vs. $0.06(0.01-0.14, \mathrm{p}=0.002]$.

Laboratory data which are relevant for the nutritional status, as well as parameters of oxidative stress and subclinical inflammation, are given at F0 and F1 in Table II. We remark a stable situation of the nutritional parameters, without decrease of total proteins, albumin or prealbumin, iron parameters, vitamins, zinc or selenium during the short nutritional intervention, as well as a tendency towards a reduction of oxidative stress, as shown by the increase in TAC $(\mathrm{p}=0.05)$. Subclinical inflammation did not progress during this short time. There was no correlation between $\gamma$-H2AX (foci/cell) and each laboratory parameter mentioned in Table II, neither at F0, nor at F1.

The number of $\gamma$-H2AX (foci/cell) was not significantly different among patients divided by gender, age, nicotine or alcohol consume or by the presence of distant metastases (Table III). The observed effect of decrease in foci number/ cell remained significantly relevant in male patients, smokers, patients without alcohol consumption and without metastases.
Since the number of patients in confounder-defined subgroups was too small to perform a multivariable analysis, we chose the subgroup comparisons, as shown above.

\section{DISCUSSION}

It is known that patients with gastrointestinal tumors are at high risk for malnutrition. We found a high nutritional risk, expressed as NRS-score $\geq 3$ in $74 \%$ of the screened patients. Of these, $82 \%$ fulfilled the criteria for malnutrition $(43 / 52)$, according to the ESPEN criteria, corresponding to $61 \%$ of the initial group [25]. Eghdam Zamiri et al. [31] found critical PG-SGA questionnaire scores $>9$ in $72.8 \%$ of patients with gastrointestinal tumors before the start of chemotherapy; poor nutritional status correlated with increased mortality. Grace et al. [32] reported that $61 \%$ of patients with oesophago-gastric cancer planned for radical treatment were moderately to severely malnourished. The combination of malignancy and malnutrition was associated with poor therapy tolerance and a 2.7 increased hazard ratio for mortality $[7,33]$.

We found increased DNA damage, measured as $\gamma$-H2AX foci in PBLCs, in patients with gastrointestinal tumors at first diagnosis and malnutrition, compared to healthy controls (Fig. 1B). Previous data pointed out to increased ratios of $\gamma-\mathrm{H} 2 \mathrm{AX}$ levels in ionized radiation treated peripheral lymphocytes to that of non-treated cells in patients with bladder cancer compared to controls, irrespective of age, sex and smoking status [16]. The $\gamma$-H2AX assay of in vitro irradiated cells revealed significant differences between patients with breast cancer and the control group with respect to post-radiation DNA damage [17]. Furthermore, nimotuzumab, a humanized IgG1 monoclonal antibody specifically targeting epidermal growth factor receptor, enhanced the radiosensitivity of lung and breast cancer cells in vitro by increasing the $\gamma$-H2AX foci and reducing DNA repair, thus favouring apoptosis [34]. In a recent study, Zhao et al. [35] described higher ratios of radiation-induced $\gamma-\mathrm{H} 2 \mathrm{AX}$ to the baseline in untreated patients with colorectal cancer compared to healthy controls, without interactions with known risk factors as age, gender, BMI or smoking status. Kvaerner et al. [36] investigated also in colorectal cancer patients whether the chemotherapyinduced genotoxicity is associated with malnutrition and found higher levels of DNA damage in blood cells of 24 patients on chemotherapy than in the remaining 20 patients without and in moderately malnourished patients than in those with good nutritional status.

Our analysis went beyond these reports. We investigated for the first time the changes in DNA damage, expressed as $\gamma$-H2AX (foci/cell), in patients with gastrointestinal tumors and $\mathrm{MN}$ at diagnosis, under an early individualized NS, during the weeks of diagnostic work-up and before the start of treatment. The short time frame was chosen because the start of any treatment (surgery, chemotherapy or radiation) would alter DNA damage and would not allow an unbiassed comparison of DNA damage between baseline and follow-up. Nevertheless, we found a significant reduction in $\gamma$-H2AX (foci/cell) from $\mathrm{F} 0$ to $\mathrm{F} 1$, when the foci values became comparable to controls. Patients with a high symptom burden had higher foci values at baseline than those suffering only from inappetence and the 
Table II. Relevant laboratory data for the nutritional status, oxidative stress and subclinical inflammation are given at baseline (F1) and at follow-up before the start of treatment (F1)

\begin{tabular}{lccc}
\hline & F0 & F1 & $\mathrm{p}$ \\
\hline Routine laboratory parameters & & & \\
Total proteins $(64-83 \mathrm{~g} / \mathrm{dl})$ & $70.38 \pm 8.09$ & $70.00(65.00-73.00)$ & 0.576 \\
Albumin $(35-50 \mathrm{~g} / \mathrm{dl})$ & $32.00(28.00-36.50)$ & $32.00(27.25-34.75)$ & 0.545 \\
Prealbumin $(0,2-0,4 \mathrm{~g} / \mathrm{dl})$ & $0.17 \pm 0.09$ & $0.18 \pm 0.08$ & 0.797 \\
Leucocytes $(3.5-10 / \mathrm{nl})$ & $7.87(6.55-11.50)$ & $7.74(5.88-12.30)$ & 0.712 \\
Lymphocytes $(16-45 \%)$ & $19.84 \pm 9.96$ & $17.88 \pm 6.73$ & 0.430 \\
Iron $(50-170 \mathrm{~g} / \mathrm{dl})$ & $62.00(42.50-95.25)$ & $54.00(44.50-78.00)$ & 0.462 \\
Ferritin $(20-275 \mathrm{ng} / \mathrm{ml})$ & $258.00(113.75-608.75)$ & $183.00(91.25-642.00)$ & 0.528 \\
Transferrin $(1.8-3.8 \mathrm{~g} / \mathrm{l})$ & $2.08(1.81-2.40)$ & $2.25 \pm 0.63$ & 0.524 \\
Transferrin saturation $(16-45 \%)$ & $22.40(15.18-29.30)$ & $21.11 \pm 10.06$ & 0.339 \\
Vitamin B12 $(190-880 \mathrm{pg} / \mathrm{ml})$ & $468.00(352.50-968.00)$ & $479.00(300.00-679.00)$ & 0.604 \\
Folic acid $(>3.1 \mathrm{ng} / \mathrm{ml})$ & $5.55(3.80-8.48)$ & $6.30(3.80-7.80)$ & 0.752 \\
Zinc $(60-120 \mu \mathrm{g} / \mathrm{dl})$ & $65.40 \pm 16.94$ & $64.37 \pm 10.41$ & 0.808 \\
Selenium $(74-139 \mu \mathrm{g} / \mathrm{l})$ & $63.22 \pm 15.46$ & $67.54 \pm 17.84$ & 0.375 \\
25-hydroxy-vitamin D $(30-80 \mathrm{ng} / \mathrm{ml})$ & $17.62 \pm 12.50$ & $19.14 \pm 12.72$ & 0.660 \\
\hline Parameters for oxidative stress & & & \\
TAC $(<200 \mu \mathrm{mol} / \mathrm{l})$ & $547.00(130.00-844.00)$ & $797.76 \pm 548.99$ & 0.056 \\
SOD $(1102-1601 \mathrm{U} / \mathrm{g} \mathrm{Hb})$ & $6711.13 \pm 1670.66$ & $5977.97 \pm 1356.38$ & 0.154 \\
\hline Parameters of subclinical inflammation & & & \\
hsCRP $(\mathrm{N}<5 \mathrm{mg} / \mathrm{l})$ & $13.00(2.40-36.00)$ & $13.80(2.52-49.40)$ & 0.824 \\
IL-6 (N=1-7 pg/ml) & $13.00(5.00-37.00)$ & $14.00(4.00-22.00)$ & 0.422 \\
PAI-1 $(\mathrm{N}=1-7 \mathrm{U} / \mathrm{ml})$ & $10.93(2.85-34.44)$ & $6.01(3.04-31.26)$ & 0.762 \\
\hline
\end{tabular}

TAC: total antioxidant capacity; SOD: superoxid-dismutase; hsCRS: high sensitive $\mathrm{C}$ reactive protein; IL-6: interleukin 6; PAI-1: plasminogen activator inhibitor 1.

foci reduction was stronger in this patient subgroup. Higher symptom burden was associated with poorer nutritional status at baseline, after 3 and 12 months in patients with oesophagogastric cancer [32]. However, there are neither literature data regarding the influence of NS on DNA damage, nor reports on the relationship of this parameter with clinical data.

Weight, BMI, laboratory parameters with relevance to the nutritional status and the subclinical inflammation remained stable during the short nutritional intervention, while TAC tendentially increased. Greater occurrence of death was observed in patients with a high SGA score, low serum albumin and increased CRP, while a positive correlation between weight loss percentage and serum CRP levels was reported [37]. There is evidence showing increased oxidative stress in tumors and multiple links between ROS and carcinogenesis. Excessive production of reactive oxygen/nitrogen species may lead to consequent alteration in the intracellular homeostasis and cause damage to all important cellular components when the

Table III. The number of $\gamma-\mathrm{H} 2 \mathrm{AX}$ foci/cell, according to gender, age, nicotine / alcohol consumption and the presence of distant metastases at baseline (F0) and at follow-up before the start of treatment $(\mathrm{F} 1)$

\begin{tabular}{lccc}
\hline & F0 & F1 & $\mathrm{p}$ \\
\hline Gender & & & \\
$\quad$ Men $(\mathrm{n}=29)$ & $0.29(0.13-0.53)$ & $0.13(0.07-0.17)$ & 0.036 \\
$\quad$ Women $(\mathrm{n}=14)$ & $0.55(0.19-1.06)$ & $0.31 \pm 0.21$ & 0.160 \\
Age & & & \\
$\quad<70$ years (n=25) & $0.44(0.15-0.89)$ & $0.16(0.11-0.56)$ & 0.133 \\
$\quad$ >70 years (n=18) & $0.24(0.15-0.69)$ & $0.11(0.08-0.22)$ & 0.105 \\
Smoker status & & & \\
$\quad$ Non-smokers (n=16) & $0.45(0.12-0.81)$ & $0.16(0.09-0.35)$ & 0.126 \\
$\quad$ Smokers (n=27) & $0.29(0.16-0.61)$ & $0.15(0.09-0.40)$ & 0.034 \\
Alcohol consumption & & & \\
$\quad$ No (n=31, $<20 \mathrm{~g} /$ day/6 months) & $0.32(0.15-0.65)$ & $0.14(0.09-0.25)$ & 0.010 \\
$\quad$ Yes (n=12, $>20 \mathrm{~g} /$ day/6 months) & $0.50(0.15-1.21)$ & $0.16(0.11-0.91)$ & 0.493 \\
Distant metastases & & & \\
$\quad$ No (n=18) & $0.27(0.14-0.58)$ & $0.16(0.07-0.29)$ & 0.041 \\
$\quad$ Yes (n=25) & $0.44(0.16-0.92)$ & $0.15(0.10-0.46)$ & 0.160 \\
\hline
\end{tabular}

Values are given as mean and standard deviation if they were normally distributed and as median and quartiles otherwise. 
excess of oxidants is not balanced by antioxidant defense due to inadequate or excessive nutrient supply and/or DNA repair mechanisms. Chronic oxidative stress can drive carcinogenesis by triggering DNA mutations, damage, and pro-oncogenic signaling, altering expression of cancer-related genes and causing mutation and transformation $[38,39]$.

Several foods and consumption patterns have been associated with various cancers and approximately 30-35\% of the cancer cases are correlated with overnutrition or malnutrition. Oxidative stress can be induced by overnutrition and obesity or by malnutrition and depends in part on nutrient quantity and quality. A human intervention study in 23 healthy, nonsmoking young men has shown that the nutritional enrichment with carotenoid containing vegetables such as tomatoes, spinach and carrots resulted in a significant decrease of strand breaks in lymphocyte DNA, while the oxidative base damage was also significantly reduced. This study suggested for the first time a protective effect of carotenoids against cancer via a decrease in oxidative stress and DNA damage in humans [24]. A further study showed a correlation between decreased mean corpuscular volume and primary DNA damage in healthy young subjects with insufficient iron intake from a low socioeconomic community [22].

In the light of these data, our finding of DNA damage decrease, even after a short individualized nutritional intervention, might be interpreted as an effect of metabolic stabilization after optimal caloric support, enriched with the necessary vitamins and minerals, pleading once more for the importance of fighting $\mathrm{MN}$ in cancer patients.

Beyond malignancy, there are several risk factors known to increase DNA damage, such as age, nicotine or alcohol consumption. Overproduction of ROS led to an increased DNA fragmentation and, consequently, to apoptosis in monocytes from normocholesterolemic old mice, which was aggravated in age-matched atherosclerotic mice [40]. Zhang et al. [41] showed that all toxicants contained in cigarettes were genotoxic in a dose- or time-dependent manner based on the in vitro $\gamma \mathrm{H} 2 \mathrm{AX}$ HCS assay, and acrolein had the strongest potential to induce DNA damage. Alcohol exposure induced elevated $\gamma \mathrm{H} 2 \mathrm{AX}$, downregulated DSB repair pathway proteins, and increased apoptotic factors in hippocampal cells of rats [21]. Laengle et al. [42] showed that $\gamma \mathrm{H} 2 \mathrm{AX}$ is a negative prognostic marker for recurrent free and overall survival in patients with colorectal liver metastases; it correlated inversely with immunogenic cell death and the associated $\mathrm{T}$ cell infiltrate. In view of these data, we analyzed the impact of possible influencing factors in our patients' group and found no relevant differences in $\gamma \mathrm{H} 2 \mathrm{AX}$ (foci/cell) according to gender, age, nicotine or alcohol consumption or by the presence of distant metastases. Male patients, smokers, patients without alcohol intake and without metastases showed a significant decrease in $\gamma \mathrm{H} 2 \mathrm{AX}$ (foci/cell) under the nutritional intervention.

A limitation of our study was that we could not realize a longer follow-up of DNA damage under nutritional intervention, since this would have been altered under confounders, represented by any treatment modality. This was a pilot monocentric study with a limited number of patients, a heterogeneous population of gastrointestinal and liver tumors and an explorative character.

\section{CONCLUSIONS}

The strength and novelty of our study was to analyze DNA damage, oxidative stress and subclinical inflammation in dynamics, in the combined setting of gastrointestinal malignancy at diagnosis and malnutrition, at baseline and after a short pretherapeutic individualized NS. We found at baseline increased $\gamma \mathrm{H} 2 \mathrm{AX}$ (foci/cell), which decreased after NS at values comparable to healthy controls. Patients with a strong gastrointestinal symptom burden had higher DNA damage but also showed a greater reduction in $\gamma \mathrm{H} 2 \mathrm{AX}$ (foci/cell) under caloric support. Clinical and nutritional laboratory parameters remained stable, while the oxidative stress tendentially decreased. The DNA damage reduction was not dependent on influencing known genotoxic factors such as gender, age, nicotine or alcohol intake or the presence of metastases. Further studies, including larger patient numbers in a multicentric setting, are required to verify these observations.

\section{Conflicts of interest: None to declare.}

Authors' contribution: A.Z. conceived and designed the study, analyzed the data, drafted the paper and critically revised the paper. B.K., P.R.G., M.M.W.: designed the study and critically revised the paper. L.M.B., E.D., H.R.: collected and analyzed the data, performed the statistical analysis, drafted and critically revised the paper. P.G., A.W., M.M., T.Z.: interpreted the data and critically revised the paper. All authors critically approved the final version to be published and agree to be accountable for all aspects of the work in ensuring that questions related to the accuracy or integrity of the work are appropriately investigated and resolved.

Acknowledgements: This work was supported by an intramural grant of the University of Mainz to A.Z. (Inneruniversitäre Forschungsförderung). The funding source was not involved in the study design, in the collection, analysis and interpretation of data, in the writing or the decision to submit the article for publication. We thank Adriana Degreif from the Institute for Clinical Chemistry and Laboratory Medicine for her excellent technical assistance with the patients' blood samples.

\section{REFERENCES}

1. Arends J, Bertz H, Bischoff C, et al; DGEM Steering Committee. S3Guideline of the German Society for Nutritional Medicine (DGEM) in Cooperation with the DGHO, the ASORS and the AKE Clinical Nutrition in Oncology. Aktuelle Ernährungsmedizin 2015;40:e1-e74 doi:10.1055/s-0035-1552741

2. Weinmann A, Braga M, Carli F, et al. ESPEN guideline: Clinical nutrition in surgery. Clin Nutr 2017;36:623-650. doi:10.1016/j. clnu.2017.02.013

3. Bozzetti F, Arends J, Lundholm K, et al; ESPEN. ESPEN Guidelines on Parenteral Nutrition: non-surgical oncology. Clin Nutr 2009;28:445-454. doi:10.1016/j.clnu.2009.04.011

4. Akbulut G. New perspective for nutritional support of cancer patients: Enteral/parenteral nutrition. Exp Ther Med 2011;2:675-684 doi:10.3892/etm.2011.247 
5. Kirkil C, Bulbuller N, Aygen E, et al. The effect of preoperative nutritional support on patients with gastrointestinal cancer:prospective randomized study. Hepatogastroenterology 2012;59:86-89.

6. Szpetnar M, Matras P, Kielczykowska M, et al. Antioxidants in patients receiving total parenteral nutrition after gastrointestinal cancer surgery. Cell Biochem Funct 2012;30:211-216. doi:10.1002/cbf.1837

7. Aaldriks AA, van der Geest LG, Giltay EJ, et al. Frailty and malnutrition predictive of mortality risk in older patients with advanced colorecta cancer receiving chemotherapy. J Geriatr Oncol 2013;4:218-226. doi:10.1016/j.jgo.2013.04.001

8. Mah LJ, El-Osta A, Karagiannis TC. GammaH2AX as a molecular marker of aging and disease. Epigenetics 2010;5:129-136. doi:10.4161/ epi.5.2.11080

9. Roos WP, Kaina B. DNA damage-induced cell death:from specific DNA lesions to the DNA damage response and apoptosis. Cancer Lett 2013;332:237-248. doi:10.1016/j.canlet.2012.01.007

10. Nikolova T, Dvorak M, Jung F, et al. The gammaH2AX assay for genotoxic and nongenotoxic agents:comparison of $\mathrm{H} 2 \mathrm{AX}$ phosphorylation with cell death response. Toxicol Sci 2014;140:103-117. doi:10.1093/toxsci/ kfu066

11. Kinner A, Wu W, Staudt C, Iliakis G. Gamma-H2AX in recognition and signaling of DNA double-strand breaks in the context of chromatin. Nucleic Acids Res 2008;36:5678-594. doi:10.1093/nar/gkn550

12. Valdiglesias V, Giunta S, Fenech M, et al. $\gamma \mathrm{H} 2 \mathrm{AX}$ as a marker of DNA double strand breaks and genomic instability in human population studies. Mutat Res 2013;753:24-40. doi:10.1016/j.mrrev.2013.02.001

13. Rothkamm K, Krueger I, Thompson LH, Löbrich M. Pathways of DNA double-strand break repair during the mammalian cell cycle. Mol Cell Biol 2003;23:5706-5715. doi:10.1128/mcb.23.16.57065715.2003

14. Graf F, Fahrer J, Maus S, et al. DNA double strand breaks as predictor of efficacy of the alpha-particle emitter Ac-225 and the electron emitter Lu-177 for somatostatin receptor targeted radiotherapy. PLoS One 2014;9:e88239. doi:10.1371/journal.pone.0088239

15. Halazonetis TD, Gorgoulis VG, Bartek J. An oncogene-induced DNA damage model for cancer development. Science 2008;319:1352-1355 doi:10.1126/science.1140735

16. Fernandez MI, Gong Y, Ye Y, et al. $\gamma$-H2AX level in peripheral blood lymphocytes as a risk predictor for bladder cancer. Carcinogenesis 2013;34:2543-254.7 doi:10.1093/carcin/bgt270

17. Djuzenova CS, Elsner I, Katzer A, et al. Radiosensitivity in breast cancer assessed by the histone gamma-H2AX and 53BP1 foci. Radiat Oncol 2013;8:98. doi:10.1186/1748-717X-8-98

18. Liu J, Wang H, Qu A, et al. Combined effects of C225 and 125-iodine seed radiation on colorectal cancer cells. Radiat Oncol 2013;8:219. doi:10.1186/1748-717X-8-219

19. Mondal NK, Sorensen E, Hiivala N, et al. Oxidative stress, DNA damage and repair in heart failure patients after implantation of continuous flow left ventricular assist devices. Int J Med Sci 2013;10:883-893. doi:10.7150/ijms.6219

20. Zhang S, Chen $\mathrm{H}$, Wang A, Liu Y, Hou H, Hu Q. Genotoxicity analysis of five particle matter toxicants from cigarette smoke based on gammaH2AX assay combined with Hill/Two-component model. Environ Toxicol Pharmacol 2018;58:131-140. doi:10.1016/j. etap.2018.01.003

21. Suman S, Kumar S, N' Gouemo P, Datta K. Increased DNA doublestrand break was associated with downregulation of repair and upregulation of apoptotic factors in rat hippocampus after alcohol exposure. Alcohol 2016;54:45-50. doi:10.1016/j.alcohol.2016.06.003
22. Pra D, Bortoluzzi A, Mueller LL, et al. Iron intake, red cell indicators of iron status, and DNA damage in young subjects. Nutrition 2011;27:293297. doi:10.1016/j.nut.2010.02.001

23. Bub A, Watzl B, Blockhaus M, et al. Fruit juice consumption modulates antioxidative status, immune status and DNA damage. J Nutr Biochem 2003;14:90-98. doi:10.1016/s0955-2863(02)00255-3

24. Pool-Zobel BL, Bub A, Müller H, Wollowski I, Rechkemmer G. Consumption of vegetables reduces genetic damage in humans:first results of a human intervention trial with carotenoid-rich foods. Carcinogenesis 1997;18:1847-1850. doi:10.1093/carcin/18.9.1847

25. Cederholm T, Bosaeus I, Barazzoni R, et al. Diagnostic criteria for malnutrition - An ESPEN Consensus Statement. Clin Nutr 2015;34:335340. doi:10.1016/j.clnu.2015.03.001

26. Reilly HM, Martineau JK, Moran A, Kennedy H. Nutritional screening-evaluation and implementation of a simple Nutrition Risk Score. Clin Nutr 1995;14:269-273. doi:10.1016/s0261-5614(95)80063-8

27. Bischoff SC, Arends J, Dörje F, et al; DGEM Steering Committee. S3Guideline of the German Society of Nutritional Medicine (DGEM) in Cooperation with the GESKES and the AKE. Nutritional Support in the Homecare and Outpatient Sector. Aktuel Ernaehrungsmed 2013;38:e101-e154. doi:10.1055/s-0033-1349549

28. Bergholz LM, Subic-Wrana C, Heylmann D, Beutel ME, Wiltink J, Kaina B. DNA damage in lymphocytes of patients suffering from complex traumatization. DNA Repair (Amst) 2017;52:103-109. doi:10.1016/j. dnarep.2017.02.006

29. Heylmann D, Kaina B. The gammaH2AX DNA damage assay from a drop of blood. Sci Rep 2016;6:22682. doi:10.1038/srep22682

30. Bauer M, Goldstein M, Christmann M, Becker H, Heylmann D, Kaina B. Human monocytes are severely impaired in base and DNA double-strand break repair that renders them vulnerable to oxidative stress. Proc Natl Acad Sci U S A 2011;108:21105-21110. doi:10.1073/pnas.1111919109

31. Eghdam Zamiri R, Salarpour F, Ghadimi Z, et al. Clinical Assessment of Malnutrition in Patients With Gastrointestinal Cancer During Chemotherapy: A Prospective Study. Rep Radiother Oncol 2015;2:e2037. doi:10.5812/rro.2(1)2015.2037

32. Grace EM, Shaw C, Lalji A, Mohammed K, Andreyev HJN, Whelan K. Nutritional status, the development and persistence of malnutrition and dietary intake in oesophago-gastric cancer:a longitudinal cohort study. J Hum Nutr Diet 2018;31:785-792. doi:10.1111/jhn.12588

33. De Divitiis C, Nasti G, Montano M, Fisichella R, Iaffaioli VR, Berretta M Prognostic and predictive response factors in colorectal cancer patients: between hope and reality. World J Gastroenterol 2014;20:15049-15059. doi:10.3748/wjg.v20.i41.15049

34. Qu YY, Hu SL, Xu XY, et al. Nimotuzumab enhances the radiosensitivity of cancer cells in vitro by inhibiting radiation-induced DNA damage repair. PLoS One 2013;8:e70727. doi:10.1371/journal.pone.0070727

35. Zhao L, Chang DW, Gong Y, Eng C, Wu X. Measurement of DNA damage in peripheral blood by the gamma- $\mathrm{H} 2 \mathrm{AX}$ assay as predictor of colorectal cancer risk. DNA Repair (Amst) 2017;53:24-30. doi:10.1016/j. dnarep.2017.03.001

36. Kvaerner AS, Minaguchi J, El Yamani N, et al. DNA damage in blood cells in relation to chemotherapy and nutritional status in colorectal cancer patients-A pilot study. DNA Repair (Amst) 2018;63:16-24. doi:10.1016/j.dnarep.2018.01.005

37. De Souza Costa MD, Vieira de Melo CY, Amorim AC, et al. Association Between Nutritional Status, Inflammatory Condition, and Prognostic Indexes with Postoperative Complications and Clinical Outcome of Patients with Gastrointestinal Neoplasia. Nutr Cancer 2016;68:11081114. doi:10.1080/01635581.2016.1206578 
38. Kruk J, Aboul-Enein HY. Reactive Oxygen and Nitrogen Species in Carcinogenesis:Implications of Oxidative Stress on the Progression and Development of Several Cancer Types. Mini Rev Med Chem 2017;17(11):904-919. doi:10.2174/1389557517666170228115324

39. Saha SK, Lee SB, Won J, et al. Correlation between Oxidative Stress, Nutrition, and Cancer Initiation. Int J Mol Sci 2017;18:1544. doi:10.3390/ijms18071544

40. Jacinto TA, Meireles GS, Dias AT, et al. Increased ROS production and DNA damage in monocytes are biomarkers of aging and atherosclerosis. Biol Res 2018;51:33. doi:10.1186/s40659-018-0182-7
41. Zhang S, Chen H, Wang A, Liu Y, Hou H, Hu Q. Assessment of genotoxicity of four volatile pollutants from cigarette smoke based on the in vitro gammaH2AX assay using high content screening. Environ Toxicol Pharmacol 2017;55:30-36. doi:10.1016/j. etap.2017.07.005

42. Laengle J, Stift J, Bilecz A, et al. DNA damage predicts prognosis and treatment response in colorectal liver metastases superior to immunogenic cell death and T cells. Theranostics 2018;8:3198-3213. doi:10.7150/thno.24699 